\title{
Optical Absorption in Commercial Single Mode Optical Fibers in a High Energy Physics Radiation Field
}

\author{
Thijs Wijnands, Luit Koert De Jonge, Jochen Kuhnhenn, Stefan Klaus Hoeffgen, Member, IEEE, and Udo Weinand
}

\begin{abstract}
This paper reports on the radiation induced attenuation of light at $1310 \mathrm{~nm}$ and $1550 \mathrm{~nm}$ in 12 commercially available single mode (SM) optical fibers. The fiber samples are exposed to gamma rays from a ${ }^{60} \mathrm{Co}$ source and to a high energy physics radiation field. The attenuation is studied as a function of total dose, dose rate, light power and temperature. Radiation hard fibers from one manufacturer show an extraordinary low attenuation for light at $1310 \mathrm{~nm}$ that does not exceed $5 \mathrm{~dB} / \mathrm{km}$ even after a total dose of 1 MGy. $2500 \mathrm{~km}$ of this type of fiber have been produced by the manufacturer and quality assurance measurements of the production batches are presently ongoing.
\end{abstract}

Index Terms-Gamma rays, high energy radiation field, industrial production, optical fibers, quality assurance, radiation induced attenuation.

\section{INTRODUCTION}

$\mathbf{O}$ PTICAL absorption in silica optical fibers has been studied in great detail over the last few decades and is usually attributed to intrinsic defects from interstitial and structural anomalies and to extrinsic defects from substitution impurities such as hydroxyl and chloride. An important difference between these two types of defects is that extrinsic anomalies can theoretically be entirely suppressed while intrinsic anomalies can only be reduced but never eliminated.

Since the 1970's the fiber manufacturing process has made enormous progress and it is now possible to produce ultrahighpurity silica $\left(\mathrm{OH}\right.$ and metal ion content below the $\mathrm{ppb}_{\mathrm{W}}$ level). In addition, the transmission window has shifted to the long wavelength region $(1310-1550 \mathrm{~nm})$ where the loss spectrum of silica glass exhibits its minimum value. This has made it possible to produce high quality step index Single Mode Fibers with an optical attenuation lower than $0.15 \mathrm{~dB} / \mathrm{km}$ at $1550 \mathrm{~nm}$ [1] which is close to the theoretical (Rayleigh) limit of transparency. When silica fibers are exposed to radiation, additional defects are generated in the silica through trapping of holes or electrons at intrinsic precursor sites and by atomic displace-

\footnotetext{
Manuscript received September 20, 2007; revised April 8, 2008. Current version published September 19, 2008.

T. Wijnands and L. K. De Jonge are with CERN, European Organization for Nuclear Research, CH-1211 Genève 23, Switzerland (e-mail: thijs.wijnands@cern.ch; luit.koert.de.jonge@cern.ch).

J. Kuhnhenn, S. K. Hoeffgen, and U. Weinand are with Fraunhofer-Institut Naturwissenschaftlich Technische Trendanalysen (INT), Appelsgarten 2 D-53879, Euskirchen, Germany (e-mail: jochen.kuhnhenn@ int.fraunhofer.de; stefan.hoeffgen@int.fraunhofer.de; udo.weinand@int.fraunhofer.de).

Digital Object Identifier 10.1109/TNS.2008.2001859
}

ments from the knock-on process [2] or the radiolysis process [3].

Extensive Electron Spin Resonance (ESR) spectrometry studies have allowed identifying various types of intrinsic paramagnetic defects in silica fibers which are E' centres [4], Non-Bridging-Oxygen-Hole-Centres (NBOHC) [5], the Peroxy Radical [6] and Self Trapped Holes [3]. Furthermore, Photo Luminescence (PL) measurements have demonstrated that some diamagnetic defects such as the Oxygen Deficiency Centre (ODC) may act as defect precursors for paramagnetic defects [7]. These colour defects have been associated with optical absorption bands at short wavelengths below $700 \mathrm{~nm}$ [8] and may already be generated during the fiber manufacturing process [9], [10].

More recently, the influence of Hydrogen and Fluorine doping on the radiation hardness has been investigated. The Hydrogen should be minimized as it may eventually create an absorption band in the long wavelength region. Under irradiation, hydrogen can react with a NBOHC (denoted as $\equiv \mathrm{Si-O}$. where the bar represents a chemical bond and the dot a dangling bond) to form an $\mathrm{OH}$ group ( $\equiv \mathrm{Si}-\mathrm{O} \cdot+\mathrm{H}_{2} \rightarrow: \equiv \mathrm{Si}-\mathrm{OH}+\mathrm{H}^{\circ}$ ). When the $\mathrm{OH}$ group in the fiber is increased in number, the optical absorption band at $1390 \mathrm{~nm}$ will increase [11]. Suitable Fluorine doping of the fiber core and cladding however can result in a higher radiation resistance in the long wavelength range as Fluorine decreases the glassy disorder and strains the $\mathrm{Si}-\mathrm{O}$ bonds in silica [12].

Despite all the knowledge available in literature, it remains uncertain how a given radiation resistant SM fiber will behave in a radiation field from high energy physics. In order to address this matter, the radiation induced attenuation (RIA) in the long wavelength region of 12 SM fiber samples from various commercial manufacturers was measured using gamma rays from a ${ }^{60}$ Co source. A description of the experimental setups is given in Section III.

After the initial screening test described in Section IV, the irradiation conditions were varied (wavelength, light power, dose, dose rate, temperature). Samples from the fibers with the lowest attenuation at 1310 and $1550 \mathrm{~nm}$ were then exposed to a high energy radiation field with particle energies up to $400 \mathrm{GeV}$. A qualitative interpretation of the experimental data is given in Section V.

\section{REQUIREMENTS}

For the application at hand [13], radiation hard SM optical fibers are required with a RIA that does not exceed $7 \mathrm{~dB} / \mathrm{km}$ 


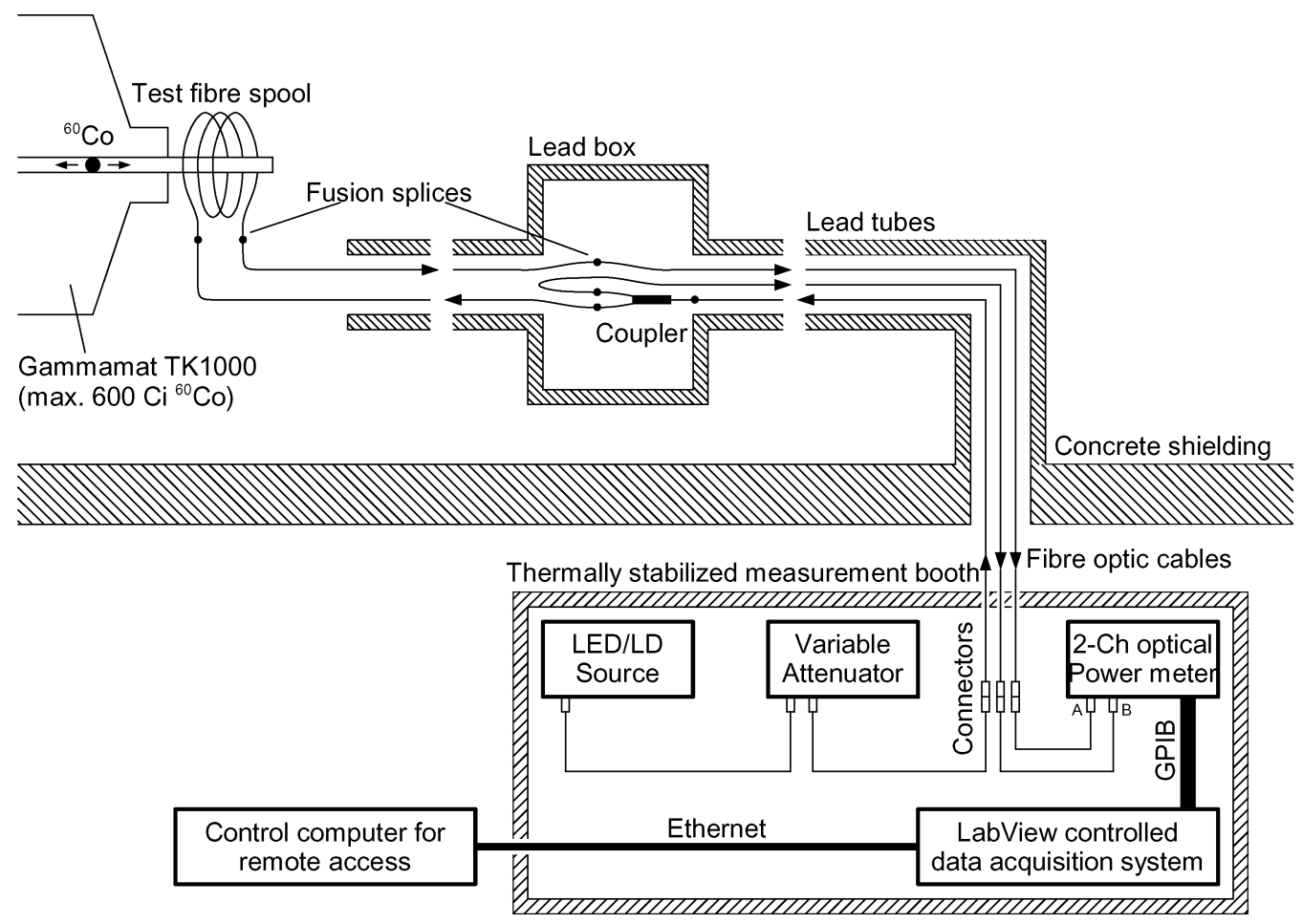

Fig. 1. Experimental setup at the ${ }^{60} \mathrm{Co}$ irradiation facility at Fraunhofer INT to measure RIA in various fiber samples on-line.

for light at $1310 \mathrm{~nm}$ after exposure to a total ionizing dose of $100 \mathrm{kGy}$ in a radiation field from high energy physics. Although there is no direct interest in the attenuation at other wavelengths than $1310 \mathrm{~nm}$, the RIA at $1550 \mathrm{~nm}$ was nevertheless considered because it may be of interest for future applications in which case it should remain of the same order of magnitude as the RIA at $1310 \mathrm{~nm}$ after $100 \mathrm{kGy}$. Only commercial fiber manufacturers with a sufficient production capacity were to be considered but the submission of production prototype samples was allowed. In total 5 of the 12 fiber samples were such development samples and are not considered further on.

The lead time for the entire operation (radiation studies, fiber manufacturing, fiber cable manufacturing, installation and commissioning) was 2 years. It was decided to allocate an entire year for the manufacturing of the fiber and fiber cable and to conduct these manufacturing activities in parallel. A period of approximately one year was available to collect samples from the manufacturers, perform the initial sample screening tests, the tests with parameter variation (wavelength, light power, temperature) and the radiation testing of the randomly chosen samples for the series production.

Final results on the RIA were then confirmed with a radiation test in a field from high energy physics. This test was considered as essential for various reasons. The first reason is that in a complex high energy physics field, radiation damage in silica fibers occurs simultaneously via the knock on process and the radiolysis process. Gamma rays from a ${ }^{60} \mathrm{Co}$ source generate mainly damage via the radiolysis effect but only a very small amount of displacement damage via secondary electrons [14]. Second, it has been reported [15]-[17] that the RIA in optical fibers from gamma rays can differ significantly from that induced by energetic neutrons. As the neutron fluence increases, the ratio of the induced loss from gamma rays and neutrons changes for the same ionising dose. When the displacement damage caused by neutrons becomes comparable to the initial defect concentration, the generation of new defects becomes the dominant effect and increase of loss with dose will be faster during neutron irradiation. For the (neutron dominated) high energy physics radiation field discussed here, the existence of such a "cross over" threshold fluence needs to be investigated. Finally, the RIA in silica fibers exposed to different types of particles at very high energies may lead to the formation of additional colour defects that have not been observed before and, given the importance of the application at hand, should not be excluded a priori.

\section{EXPERIMENTAL}

\section{A. Gamma Ray Irradiation Facility}

All gamma irradiation tests have been carried out with a calibrated ${ }^{60} \mathrm{Co}$ source at Fraunhofer INT (TK1000 Gammamat) in accordance with the IEC 60793-1-54 specifications [18] and at room temperature $\left(24{ }^{\circ} \mathrm{C}-28{ }^{\circ} \mathrm{C}\right)$. The light from the laser diode light source (LD Profile 1310) is divided by a coupler to a reference and measurement channel (Fig. 1). The reference channel compensates possible drifts of the light source. The fiber samples are wound up on aluminium spools to assure homogenous irradiation by the point source in the centre of the spool. The light transmitted via the fiber samples and via the reference channel is measured with a high precision dual channel optical power meter (HP 8153). Lead tubes avoid the exposure of the fiber cables in the vicinity of the source.

Before each irradiation, the system stability in terms of noise and drift is verified. During irradiation, the noise and drift observed via the reference channel was always below $1 \%$ of the 


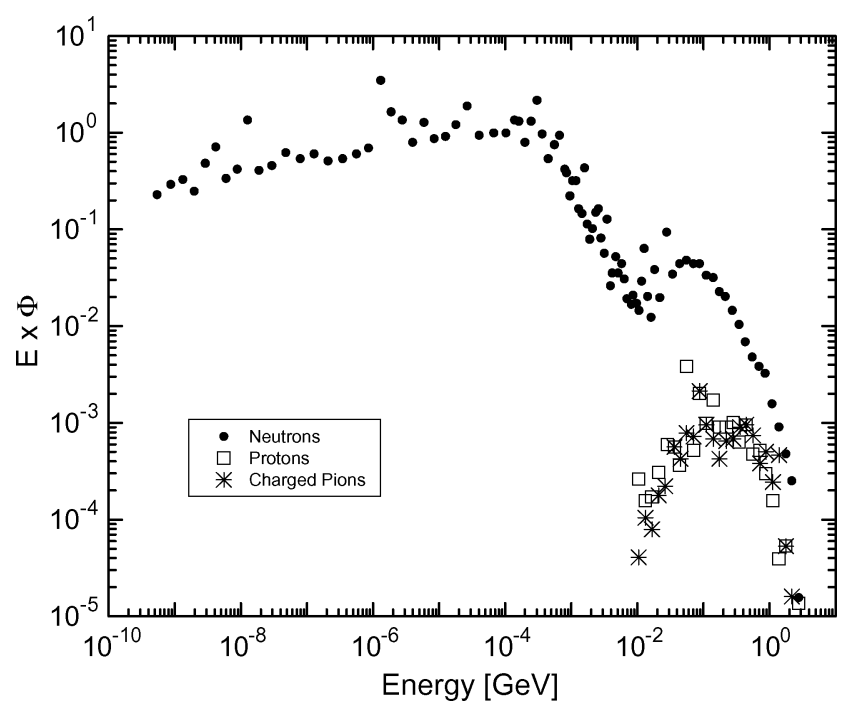

Fig. 2. Lethargy plot of the energy spectrum in the high energy radiation test facility at CERN. Normalization is to $100 \mathrm{keV}$ neutrons.

total induced loss. By varying the length of the samples, the total induced loss after irradiation in each sample was kept between 2 and $5 \mathrm{~dB}$. By limiting the total attenuation in the samples during irradiation, a good compromise is found between the signal to noise ratio in the measurements on the one hand and the light power of the measurement channel on the other to reduce photobleaching effects during the irradiation. It was later confirmed that none of the fibers investigated here showed a high sensitivity to photobleaching, see also Section IV.

The total uncertainty in the optical absorption measurements presented here is estimated to be below 10\% (not taking possible temperature effects into account).

\section{B. High Energy Physics Radiation Facility}

Irradiation of fiber samples in a high energy field were carried out in the radiation test facility of the Super Proton Synchrotron (SPS) at CERN, the European Laboratory for Particle Physics. In the SPS, fixed target beams are accelerated from $14 \mathrm{GeV} / \mathrm{c}$ to $450 \mathrm{GeV} / \mathrm{c}$ in 3 seconds and then dumped on various primary targets during a 5 second long extraction procedure. This process is repeated every 14.4 seconds 24 hours per day and 7 days per week. The high energy protons cause harmonic showers in the targets which create a pulsed radiation field in the test facility situated behind one of the primary targets. During normal operation, the averaged dose rate in the area is $5 \mathrm{~Gy} / \mathrm{d}^{1}$ at the position of the samples up to the total dose of 512 Gy. Later the samples were moved closer to the target and the mean dose rate reached $23 \mathrm{~Gy} / \mathrm{d}$. The radiation spectrum [19], [20] in the area is neutron dominated and the maximum neutron energy can reach several GeVs (Fig. 2). Apart from neutrons, the Electro Magnetic (EM) showers of the incoming high energy protons also lead to the production of lower energy protons, kaons, pions, electrons, muons and gamma rays at various energies.

The dose rate at the fiber samples in this facility is measured on line with various types of ionization chambers [21] and a

\footnotetext{
${ }^{1}$ Throughout this paper Gy means $\mathrm{Gy}\left(\mathrm{SiO}_{2}\right)$.
}

remote radiation monitoring system using Radiation Sensing Mosfets (RADFETs) [22].

Two independent systems were used to measure the RIA in the fiber samples on line during the irradiation test (Fig. 3). The first system is based on a planar wave guide system to distribute light of a very stable LED light source with cable leads to the fiber samples in the irradiation zone. The light is guided back and multiplexed with a high precision micro-electro-mechanical switch to an optical power meter.

The second system is using a direct measurement with an Optical Time Domain Reflectometer (OTDR) connected to the same micro-electro-mechanical switch. A second independent measurement was needed as a back-up solution to assure a reliable long term loss measurement. The results from both systems were in excellent agreement and are therefore not shown separately here.

The fiber samples had a length between $2 \mathrm{~km}$ and $10 \mathrm{~km}$ for this setup. During the test, all components of the test setup are shielded from EM radiation and operated at a constant temperature.

\section{RESULTS}

\section{A. Sample Screening Test $\left({ }^{60}\right.$ Co Source)}

Thirteen companies were contacted and invited to participate in the radiation screening. Each manufacturer was given the specific details of the radiation tests and confirmed that large quantities of fiber could be delivered if required. Eventually 7 fiber samples from 6 different manufacturers were used in the screening test (with figure designations): three Ge-doped silica fibers (1) (2) (3), two Pure Silica Core (PSC) fibers (4) (5), and two F-doped fibers (6) (7). These fibers have similar optical attenuation for light at $1310 \mathrm{~nm}$ before irradiation. Exemplarily Fig. 4 shows the intrinsic attenuation of the F-doped fiber (7).

All samples were exposed to gamma rays under near identical experimental conditions: wavelength $1310 \mathrm{~nm}$, total dose: 10 $\mathrm{kGy}$, dose rate: $0.2 \mathrm{~Gy} / \mathrm{s}$, temperature: $24{ }^{\circ} \mathrm{C}$ to $28{ }^{\circ} \mathrm{C}$ and light power: $10 \mu \mathrm{W}$. Only for one specific sample, the light power was increased from $10 \mu \mathrm{W}$ to $40 \mu \mathrm{W}$ to improve the stability of the measurement chain. It was later verified experimentally that photo bleaching effects in this specific fiber sample are negligible and that the variation of light power at $1310 \mathrm{~nm}$ even by a factor of more than 20 had no influence on the measurements. The sample length varied between 50 and $200 \mathrm{~m}$.

Fig. 5 shows the induced loss in $\mathrm{dB} / \mathrm{km}$ as a function of the total dose for each of the fiber samples. The Ge-doped fibers show the highest induced losses, as expected. There is remarkable difference in performance amongst the Ge-doped fibers from the various manufacturers and the RIA varies by as much as $40 \%$ in agreement with similar studies [23].

The PSC fibers show significantly lower losses at the same dose which is also in agreement with previous experimental observations [24]. The performance of the F-doped fiber (6) is considerably better compared to the PSC fiber samples, reaching 10 $\mathrm{dB} / \mathrm{km}$ after a total dose of $10 \mathrm{kGy}$. At this stage however, there is no sign of saturation of the RIA. 


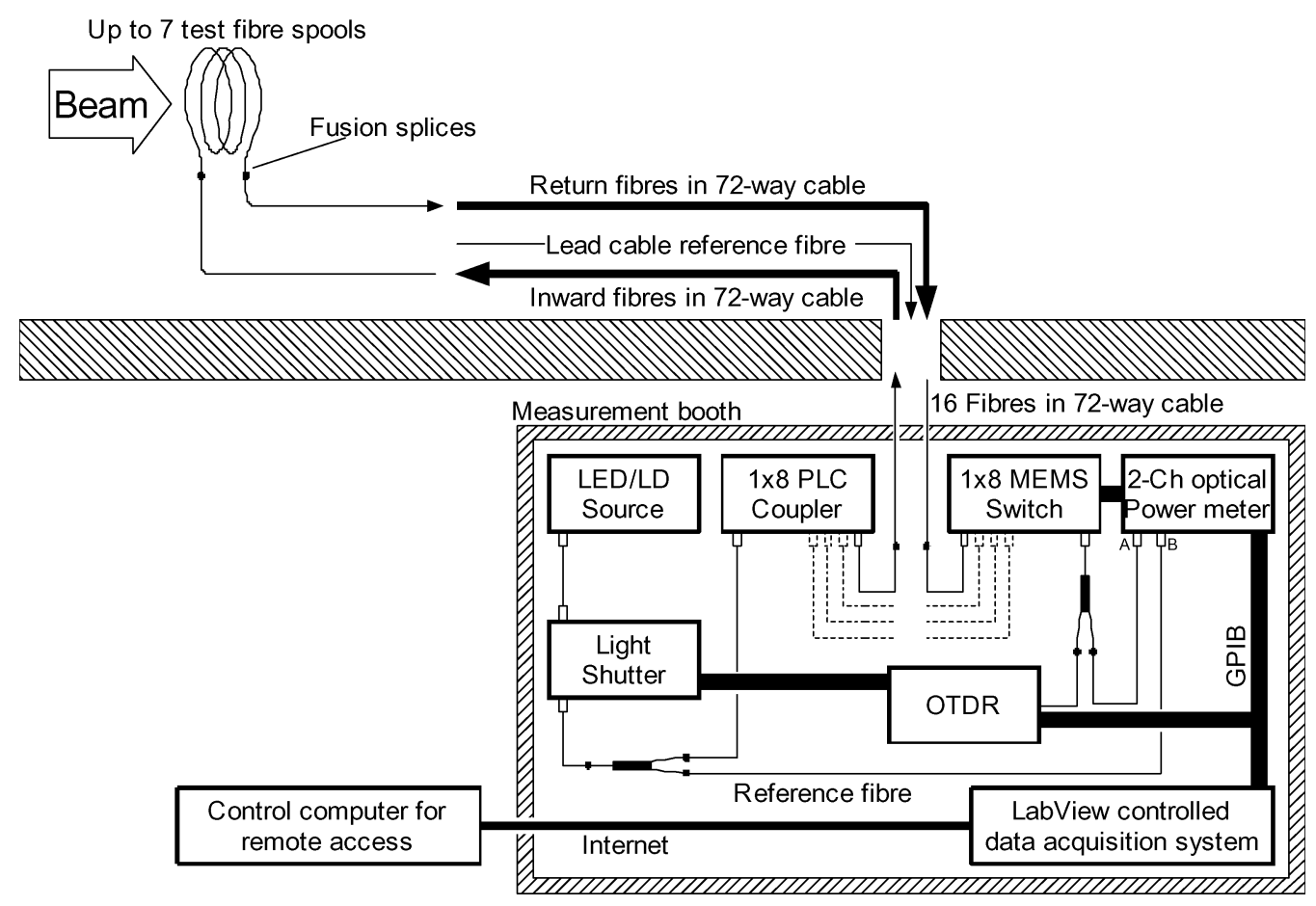

Fig. 3. Experimental setup to measure RIA in various fiber samples on-line (high energy radiation field of the Super proton Synchrotron at CERN).

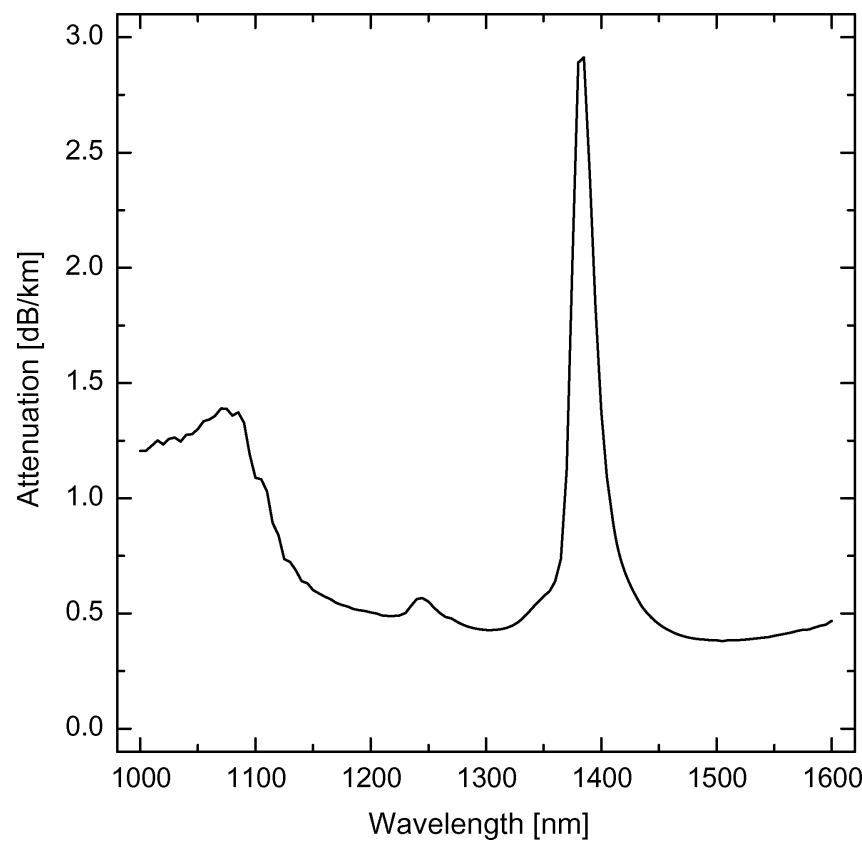

Fig. 4. Attenuation spectrum of the un-irradiated F-doped SM core fiber (7).

The F-doped fiber sample (7) has an entirely different behaviour. The loss rapidly saturates in the early stage of irradiation at approximately $2 \mathrm{~dB} / \mathrm{km}$ which is a remarkably low value in comparison with the other fiber samples.

The annealing relative to the final induced loss of the fiber samples at room temperature is shown in Fig. 6 for a period up to 100000 seconds after the end of the ${ }^{60} \mathrm{Co}$ irradiation. In the Ge-doped and the PSC fibers, a large fraction of the radiation induced losses anneal out. This provides no substantial

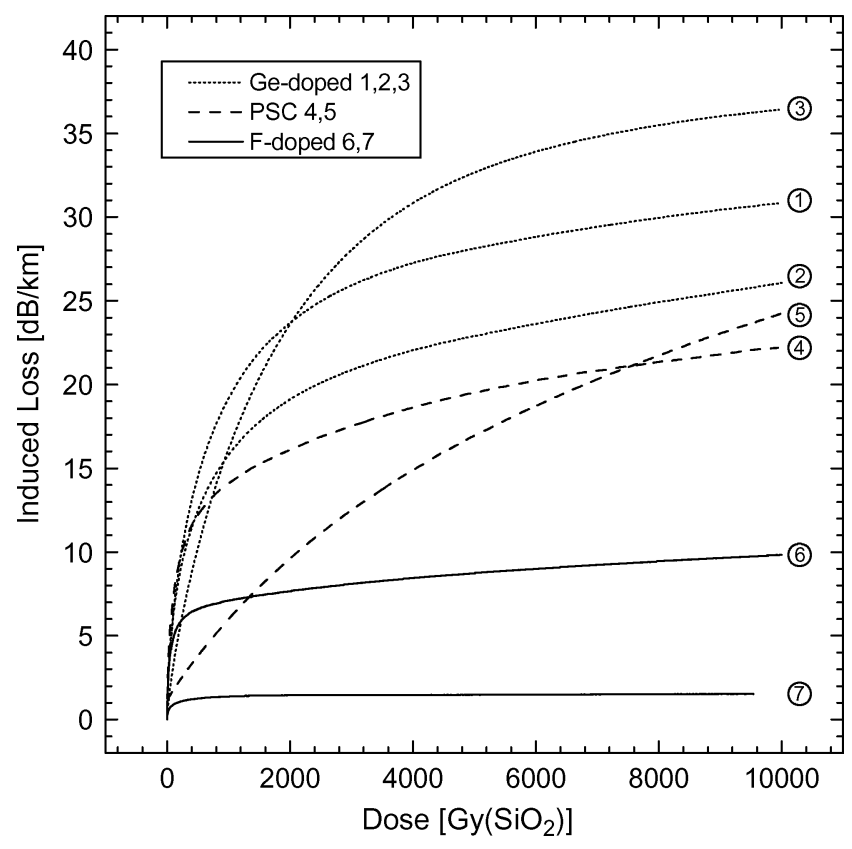

Fig. 5. RIA in the initial sample screening test $\left({ }^{60} \mathrm{Co}\right.$ source $)$.

gain however, because the attenuation rapidly attains the value before annealing once irradiation is restarted (memory effect). This has also been experimentally confirmed.

The annealing behaviour in the F-doped fiber (7) is very different from that observed in the other fiber samples. Immediately after the irradiation is stopped, this F-doped fiber exhibits the fastest annealing rate, annealing nearly $20 \%$ of the total loss in only a few seconds. The annealing is so fast that only 2 data points could be recorded which explains the discontinuity in 


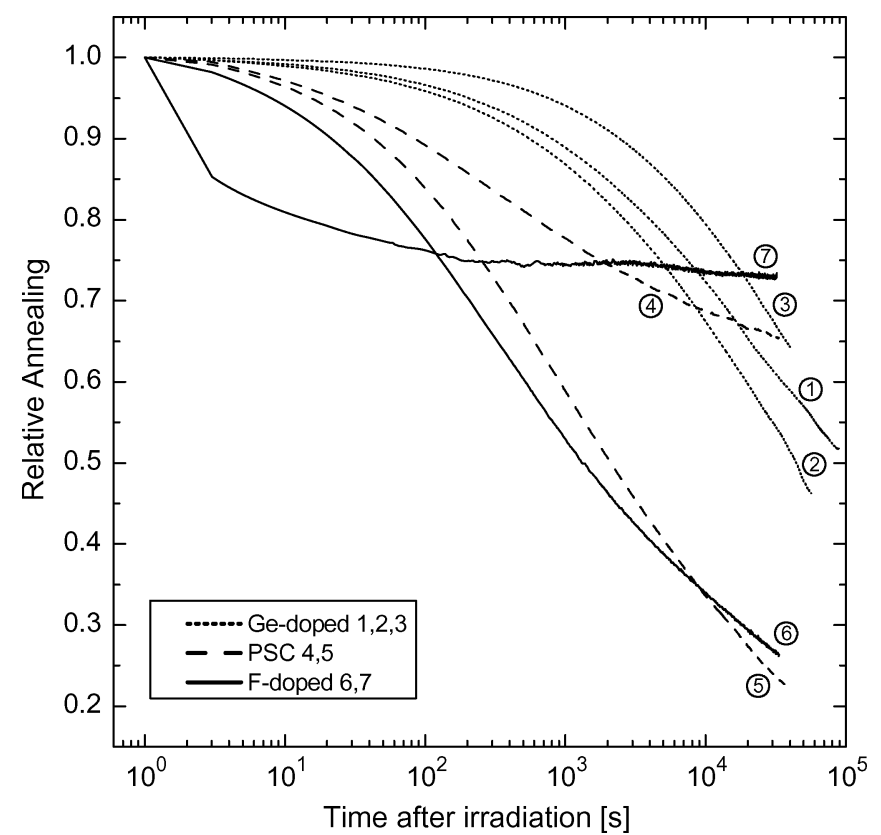

Fig. 6. Annealing (normalized to total induced loss) of the RIA after gamma ray irradiation.

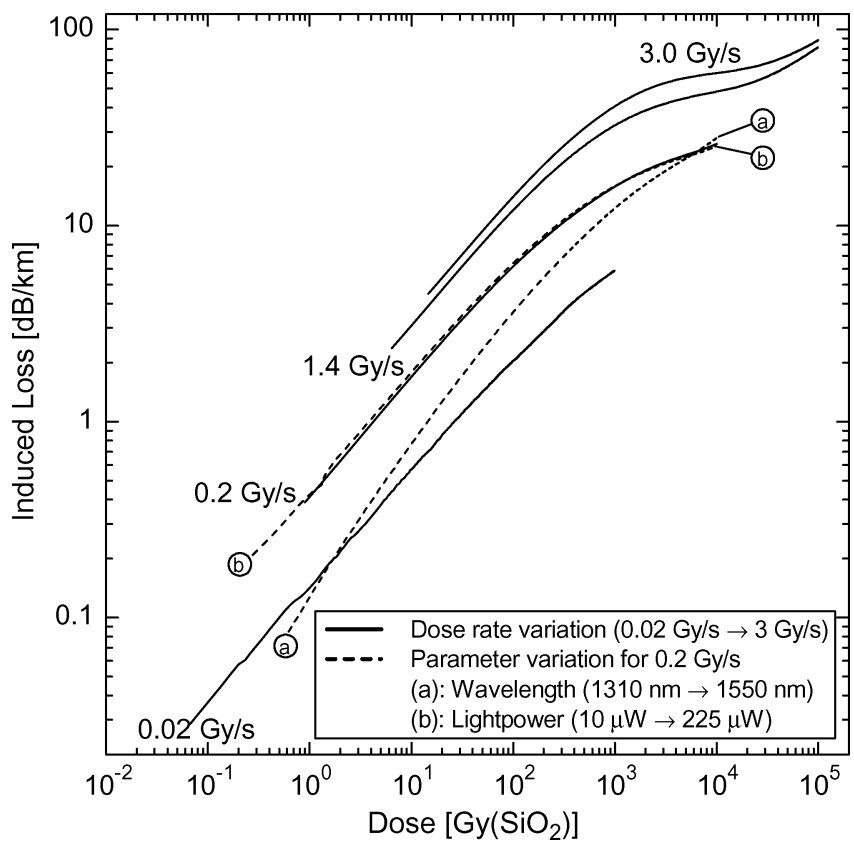

Fig. 7. Dependence of the RIA on irradiation conditions (Ge-doped fiber (2)).

the annealing curve in Fig. 6. After 100 seconds, the RIA does not reduce any further. Eventually, only $25 \%$ of the loss in this F-doped anneals at room temperature. In the other fiber samples, at least $35 \%$ of the RIA anneals after 100000 seconds.

\section{B. Variation of Irradiation Conditions $\left({ }^{60} \mathrm{Co}\right.$ Source)}

The impact of a variation of the wavelength, light power, temperature and dose rate on the RIA was investigated for the best performing Ge-doped fiber (2) (Fig. 7) and for the F-doped fiber (7) (Fig. 8). This F-doped fiber manufactured by Fujikura Ltd.

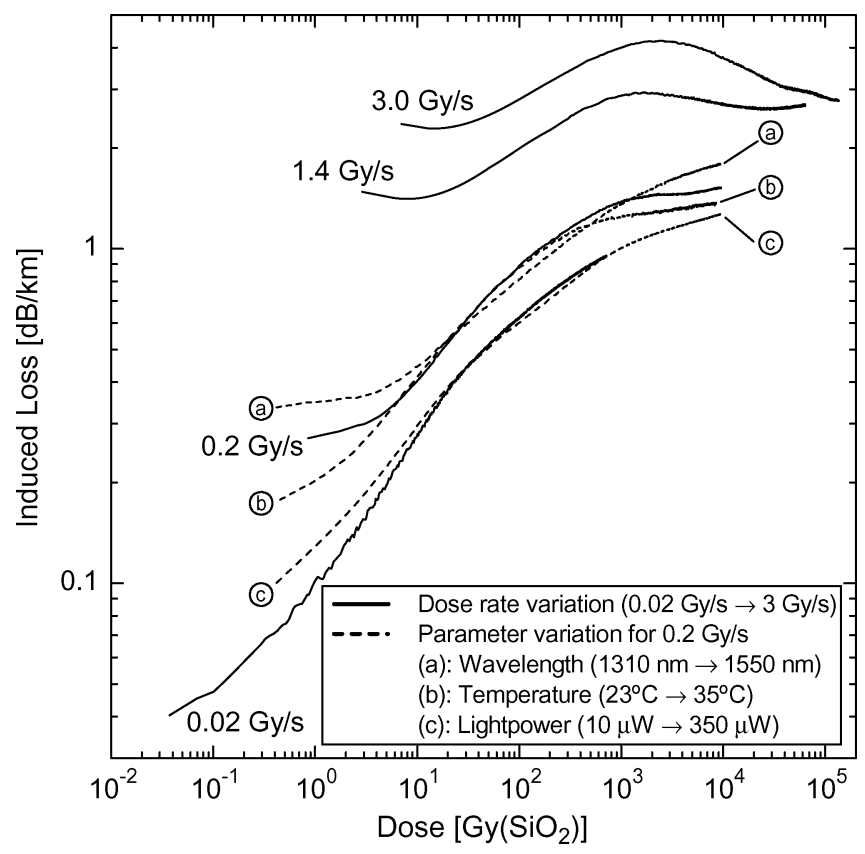

Fig. 8. Dependence of the RIA on irradiation conditions (F-doped fiber (7)).

Japan was selected because it exhibited the best performance in the initial screening test. The reason for equally investigating the Ge-doped SM fiber (2) is that this fiber is extensively used for other applications at CERN [25]. This fiber is a standard SM communication fiber produced by Draka Comteq Optical fiber BV in the Netherlands who also manufactured the F-doped fiber (6).

The dependence of the loss on wavelength, temperature and light power was investigated because it is of interest for future applications that may use the same fiber infrastructure. For the application at hand here, however, all these parameters are fixed and not subject to any change.

The dose rate dependence of the loss at $1310 \mathrm{~nm}$ is of particular importance as it allows scaling the experimental results from the test facilities to the final application.

For both the Ge-doped fiber sample (2) and the F-doped fiber sample (7), the dose rate has a large impact on the total RIA for a given dose while the influence of temperature, wavelength and light power is at most a few percent.

The impact of the dose rate on the RIA at a given dose has been investigated in detail in [26] for the Ge-doped fibers. The relaxation of the Ge-doped fiber presented here indeed follows the same 'universal' power law for diffusion controlled reactions from random distributions in the transport parameters that govern transport in disordered solids such as silica.

The behaviour of this F-doped fiber under varying irradiation conditions is again very different compared to the Ge-doped fiber. The loss seems to converge to the same value of approximately $2 \mathrm{~dB} / \mathrm{km}$ independent of the irradiation condition. This indicates that the radiation effects in the F-doped fiber are belonging to another class in which hierarchically limited dynamics lead to correlated relaxation processes that consist of several steps [26]. The observed overall behaviour is the 


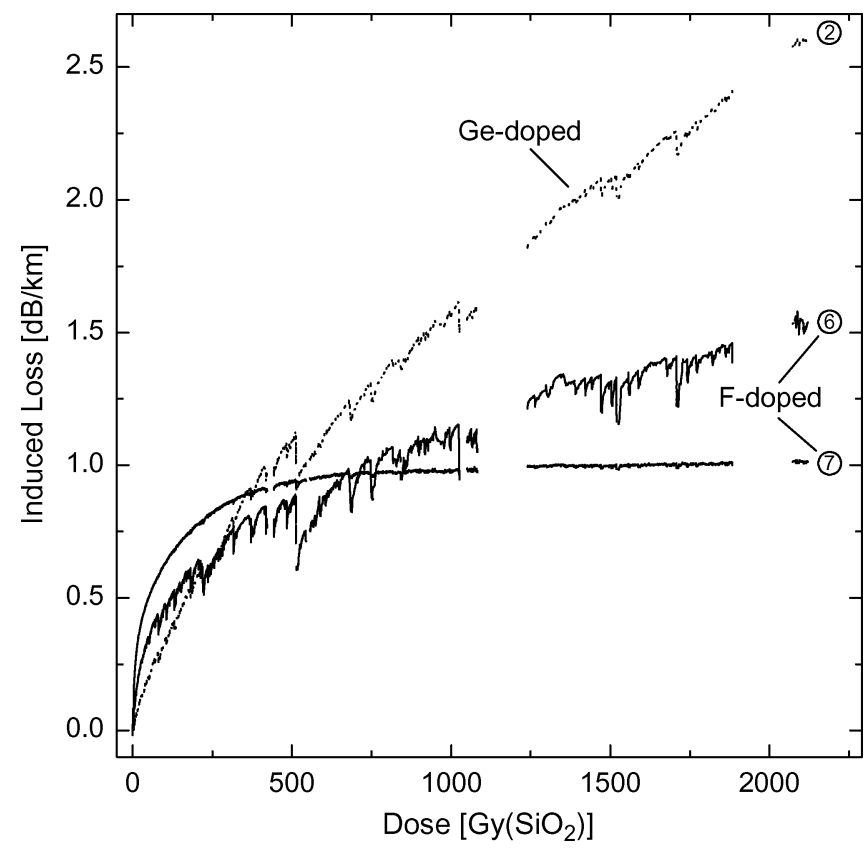

Fig. 9. RIA of the fiber samples in a high energy physics radiation field.

ensemble of several relaxation processes, each with their proper relaxation constants.

\section{Exposure to a High Energy Radiation Field}

Fig. 9 shows the induced losses in the Ge-doped fiber (2) and in the F-doped fibers as a function of the total dose in the high energy physics radiation field (see Section III). The data was accumulated in a total time span of 1.5 years. The occasional annealing in the loss curves is due to stops of the accelerator for maintenance or repairs. After a total dose of approximately $500 \mathrm{~Gy}$, the accelerator was shut down for a period of 6 months and the samples were moved closer to the target.

The induced losses in the high energy physics radiation field show a similar evolution as function of the total dose as observed during the ${ }^{60} \mathrm{Co}$ irradiation. This is in contrast to the observations in [17] where the permanent loss from neutron irradiation in SM F-doped fibers was found to be larger compared to gamma ray irradiation. The losses in the F-doped fiber (7) are very little influenced by annealing. It shows the same saturating behaviour as observed during ${ }^{60} \mathrm{Co}$ irradiation tests in which a saturated value of approximately $1 \mathrm{~dB} / \mathrm{km}$ was reached.

The period of annealing around $500 \mathrm{~Gy}$ is clearly visible for the F-doped fiber (6) and that with a Ge-doped core, whereas the other F-doped fiber does not show any annealing in this scale. For all samples the attenuation quickly reaches again the previous levels.

In Fig. 10, the RIA attenuation in the Ge-doped fiber and the F-doped fibers in a high energy physics radiation field (dose rate $5 \mathrm{~Gy} / \mathrm{d}$ up to $512 \mathrm{~Gy}$, thereafter $23 \mathrm{~Gy} / \mathrm{d}$ ) is compared to that observed during ${ }^{60} \mathrm{Co}$ irradiation (dose $1.8 \mathrm{kGy} / \mathrm{d}$ ).

For the Ge-doped fiber, the difference in absolute attenuation can be explained by the difference in dose rate between the two experimental environments. After the initial onset of the loss,

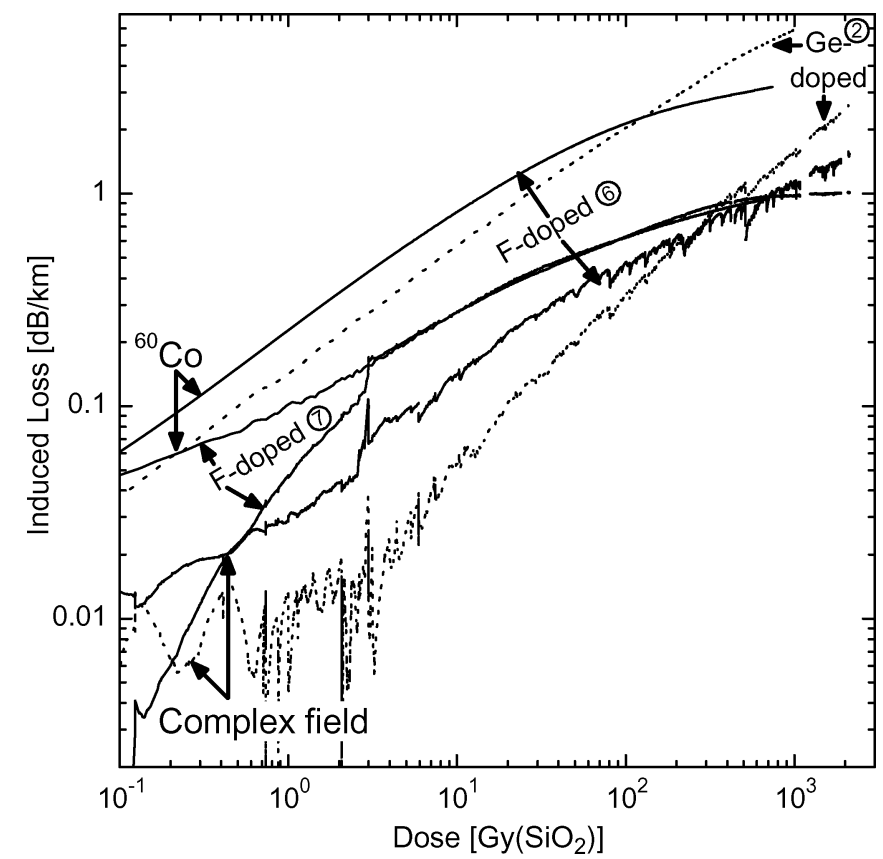

Fig. 10. Comparison of the RIA from gamma rays $(1.8 \mathrm{kGy} / \mathrm{d})$ and from a high energy physics radiation field $(5 \mathrm{~Gy} / \mathrm{d})$.

the attenuation in the F-doped fiber (7) is again converging to the same saturated value of approximately $1 \mathrm{~dB} / \mathrm{km}$, independent of the dose rate and the total dose.

The remarkable fluctuations around $3 \mathrm{~Gy}$ are due to a period of very high dose rate that led to the rapid increase of the induced loss for the F-doped fiber (7) as already observed in the ${ }^{60} \mathrm{Co}$ irradiations.

\section{DISCUSSION}

The F-doped SM optical fiber (7) from Fujikura Ltd Japan presented here exhibits a very unusual behaviour under irradiation as compared to the standard Ge-doped communication fibers and the PSC fiber samples. The RIA in this fiber for light at $1310 \mathrm{~nm}$ saturates after a total dose of approximately $1 \mathrm{kGy}$ and does not exceed $5 \mathrm{~dB} / \mathrm{km}$ for light at $1310 \mathrm{~nm}$.

Both the loss and the annealing data are suggesting that hierarchically ordered transport processes dominate in the early stages of irradiation and annealing. Although details of the manufacturing process have to remain confidential, it is evident that the manufacturing process for the Fujikura F-doped fiber has been optimized to reduce the presence of drawing induced defects in the first place. The rapid increase of the attenuation that is observed at low total doses may therefore correspond to the population of initial defects generated by the manufacturing process. In this case, the unusual annealing behaviour would be explained by a rapid depopulation of the defects in the first few seconds after the irradiation.

At higher doses, no additional absorption bands in the long wavelength region have been observed in this F-doped fiber. It was experimentally observed that the saturated loss in the F-doped fiber is strongly influenced by the $\mathrm{OH}$ content of the pristine fiber. The lower the $\mathrm{OH}$ content, the lower the value of the saturated loss. Furthermore, the doping concentration of 
the core and cladding plays an important role, perhaps by inhibiting the growth of the overtone and combination bands from the residual $\mathrm{OH}$ that is dominant at longer wavelengths. A microprobe analysis of the fiber indeed revealed a low concentration of $\mathrm{F}$ in the fiber core and very high concentration of $\mathrm{F}$ in the fiber cladding. A complete spectral attenuation study could make this issue more precise.

No significant differences between the RIA from gamma rays or from a high energy physics radiation field have been observed. In particular, the existence of a fluence threshold, after which neutron irradiation will cause a higher loss as compared to gamma rays, was not observed. We also conclude that the dominant radiation effect for optical loss in a high energy physics radiation field is the radiolysis effect and that displacement damage effects play a minor role. Finally, the various high energy physics particles at very high energies $(\mathrm{GeV}$ range) do not create new defects that cause absorption bands in the long wavelength region between 1300 and $1550 \mathrm{~nm}$.

However, it should be noted that these results are currently only based on the data obtained up to a dose of $2000 \mathrm{~Gy}$.

\section{CONCLUSION}

A comprehensive survey of the radiation tolerance of present day commercially available SM optical fibers has been conducted. The F-doped radiation resistant SM fiber from Fujikura Ltd. shows exceptionally good radiation tolerance under gamma radiation and in a high energy physics radiation field. This fiber fully meets the specifications outlined in Section II and recently, the production of 2500 fiber kilometres has been completed.

The behaviour of the fiber under irradiation was studied under various irradiation conditions using measurements of the attenuation of light. In all cases, a saturating loss curve was observed with a final value less then $5 \mathrm{~dB} / \mathrm{km}$ in line with the specifications. The measurements presented here do not allow a complete understanding of the underlying mechanisms but it was experimentally observed that the manufacturing conditions, the doping concentrations and the $\mathrm{OH}$ content are amongst key factors. A complete spectrum analysis combined with a Photoluminescence and ESR measurements could perhaps make the understanding of the underlying radiation effects more complete.

\section{ACKNOWLEDGMENT}

The authors would like to thank Dr. H. Henschel who kindly shared his long experience in the field of radiation tolerant fiber optics. They would also like to thank the following manufactures of optical fibers for their collaboration and their contributions and, in particular, for making available the various fiber samples that were used in these radiation tests: Corning, Draka Comteq Optical fiber N.V., Fujikura, Ltd., Japan, Heraeus, j-fiber, and Sumitomo.

\section{REFERENCES}

[1] K. Nagayama, M. Matsui, M. Kakui, T. Saitoh, K. Kawasaki, H. Takamizawa, Y. Ooga, I. Tsuchiya, and Y. Chigusa, "Ultra low loss $(0.1484 \mathrm{~dB} / \mathrm{km})$ pure silica core fiber," SEI Tech. Rev., no. 57, pp. 3-6, Jan. 2004.
[2] D. L. Griscom, "Self-trapped holes in amorphous silicon dioxide," Phys. Rev. B, vol. 40, no. 6, pp. 4224-4227, Aug. 1989.

[3] J. P. Vigouroux, J. P. Durand, A. L. Moel, C. L. Gressus, and D. L. Griscom, "Electron trapping in amorphous $\mathrm{SiO}_{2}$ studied by charge buildup under electron bombardement," J. Appl. Phys., vol. 57, no. 12, pp. 5139-5144, Jun. 1985.

[4] R. A. Weeks, "Paramagnetic resonance of lattice defects in irradiated quartz," J. Appl. Phys., vol. 27, no. 11, pp. 1376-1381, Nov. 1956.

[5] M. Stapelbroek, D. L. Griscom, E. J. Friebele, and G. H. Sigel, Jr., "Oxygen-associated trapped-hole centers in high-purity fused silicas," J. Non-Cryst. Sol., vol. 32, no. 1-3, pp. 313-326, Feb./Mar. 1979.

[6] E. J. Friebele, D. L. Griscom, M. Stapelbroek, and R. A. Weeks, "Fundamental defect centers in glass: The peroxy radical in irradiated, highpurity, fused silica," Phys. Rev. Lett., vol. 42, no. 20, pp. 1346-1349, May 1979.

[7] A. N. Trukhin, "Radiation processes in oxygen-deficient silica glasses: Is ODC(I) a precursor of E' center?," J. Non-Cryst. Sol., vol. 352, no. 28-29, pp. 3002-3008, Aug. 2006.

[8] D. L. Griscom, "Optical properties and structure of defects in silica glass: Optical materials," J. Ceram. Soc. Jpn., vol. 99, no. 1154, pp. 923-942, Oct. 1991.

[9] H. Hanafusa, Y. Hibino, and F. Yamamoto, "Formation mechanism of drawing-induced E centers in silica optical fibers," J. Appl. Phys., vol. 58, no. 3, pp. 1356-1361, Aug. 1985.

[10] Y. Hibino and H. Hanafusa, "Defect structure and formation mechanism of drawing-induced absorption at $630 \mathrm{~nm}$ in silica optical fibers," J. Appl. Phys., vol. 60, no. 5, pp. 1797-1801, Sep. 1986.

[11] K. Sanada and T. Kakuta, "Method of manufacturing radiation-resistant optical fiber," U.S. Patent 5269 825, Dec. 14, 1993.

[12] K. Sanada, N. Shamoto, and K. Inada, "Radiation resistance of fluorine-doped silica-core fibers," J. Non Cryst. Sol., vol. 179, no. 4, pp. 339-344, Nov. 1994.

[13] E. Effinger, B. Dehning, J. Emery, G. Ferioli, and C. Zamantzas, "Single gain radiation tolerant LHC beam loss acquisition card," presented at the 8th European Workshop on Beam Diagnostics and Instrumentation for Particle Accelerators (DIPAC), Venice, Italy, May 20-23, 2007, Paper WEPC06, unpublished.

[14] G. P. Summers, E. A. Burke, P. Shapiro, S. R. Messenger, and R. J. Walters, "Damage correlations in semiconductors exposed to gamma, electron and proton radiations," IEEE Trans. Nucl. Sci., vol. 40, no. 6, pt. 1-2, pp. 1372-1379, Dec. 1993.

[15] H. Henschel, O. Kohn, W. Lennartz, S. Metzger, H. U. Schmidt, J. Rosenkranz, B. Glessner, and B. R. L. Siebert, "Comparison between fast neutron and gamma irradiation of optical fibers," IEEE Trans. Nucl. Sci., vol. 45, no. 3, pt. 3, pp. 1543-1551, Jun. 1998.

[16] D. W. Cooke, B. L. Bennett, and E. H. Farnum, "Optical absorption of neutron-irradiated silica fibers," J. Nucl. Mater, vol. 232, no. 2-3, pp. 214-218, Sep. 1996.

[17] T. Kakuta, T. Shikama, and M. Narui, "Neutron irradiation effects on optical fibers," Sci Rep. RITU A40 (1994) No. 1, Tohoku Univ., Japan, Jul. 1994, pp. 153-157.

[18] Optical fibers-Part 1-54: Measurement Methods and Test Procedures-Gamma Irradiation, Int. Std. IEC 60793-1-54, 2003.

[19] C. A. Fynbo, Qualification of the Radiation Environment in the TCC2 Experimental Test Area CERN. Geneva, Switzerland, CERN-LHCProject-Note-235, 2000

[20] E. Tsoulou, T. Wijnands, C. Pignard, and R. Rausch, "Monte Carlo simulations of the complex field in the LHC radiation test facility at CERN," in Proc. 7th Eur. Conf. Radiation and Its Effects Components and Systems (RADECS), Noordwijk, Netherlands, 2003, pp. 601-605.

[21] C. Theis, D. Forkel-Wirth, D. Perrin, S. Roesler, and H. Vincke, "Characterisation of ionisation chambers for a mixed radiation field and investigation of their suitability as radiation monitors for the LHC," $R a$ diat. Protect. Dosimetry, vol. 116, no. 1-4, pp. 170-174, Dec. 2005.

[22] A. Jaksic, Y. Komoto, A. Mohammadzadeh, and W. Hajdas, "RADFET response to proton irradiation under different biasing configurations," in Proc. 8th Eur. Conf. Radiation and Its Effects on Components and Systems (RADECS), Cap d'Agde, France, 2005, pp. 13/1-13/5.

[23] J. Troska, J. Batten, K. A. Gill, and F. Vasey, "Radiation effects in commercial off-the-shelf single-mode optical fibers," in Proc. SPIE-Photonics for Space Environments IV, 1998, vol. 3440, pp. 112-119.

[24] H. Henschel, J. Kuhnhenn, and U. Weinand, "Radiation hard optical fibers," in Proc. Optical Fiber Commun. Conf., Tech. Dig. (OFC/NFOEC), 2005, vol. 4, p. 4.

[25] T. Freeman, "Particle accelerator gets wired up," FiberSystems Europe in Association With Lightwave Europe, p. 9, Jul./Aug. 2005.

[26] D. L. Griscom, M. E. Gingerich, and E. J. Friebele, "Radiation-induced defects in glasses: Origin of power-law dependence of concentration on dose," Phys. Rev. Lett., vol. 71, no. 7, pp. 1019-1022, Aug. 1993. 